\title{
Inkjet printable ZnO/PEDOT:PSS heterojunction for thin flexible semi-transparent optoelectronic sensors
}

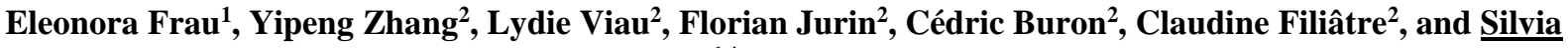 \\ Schintke $^{1, *}$, Member, IEEE \\ ${ }^{l}$ Laboratory of Applied NanoSciences, Department of Industrial Technologies, HEIG-VD, HES-SO // University \\ of Applied Sciences Western Switzerland (HES-SO), CH-1401 Yverdon-les-Bains, Switzerland \\ Tel: +41 2455761 67, e-mail: silvia.schintke@heig-vd.ch \\ ${ }^{2}$ Institut UTINAM, UMR 6213 CNRS-UBFC, Université de Bourgogne Franche-Comté (UBFC), F-25030 \\ Besançon Cedex, France
}

\begin{abstract}
Flexible sensors play an increasing role in printed electronics and are of interest for optoelectronic applications in flexible robotics and industrial automation. Thus, we have investigated the hybrid inorganic-organic junction between $\mathrm{ZnO}$ and PEDOT:PSS (poly(3,4-ethylenedioxythiophene) polystyrene sulfonate). A thin ITO (indium tin oxide) layer on PET (polyethylene terephthalate) foils was used as substrate electrode. $\mathrm{ZnO}$ was deposited from a nanoparticle (NP) suspension by electrophoretic deposition. For comparison, we have used three different methods for the deposition of PEDOT:PSS, namely (i) drop casting, (ii) dip-coating, (iii) inkjet printing. For the resulting ZnO-PEDOT:PSS junctions, current-voltage characteristics were recorded using miBot micromanipulators, and analysed applying adapted models of thermionic emission, according to Cheung and Cheung, as well as Norde. For dip-coated samples we obtained barrier heights of $(0.78 \pm 0.01) \mathrm{eV}$ and $(0.73 \pm 0.02) \mathrm{eV}$, respectively, while inkjet printed and drop casted structures show smaller values $(0.53 \mathrm{eV}$ to $0.66 \mathrm{eV}$, respectively). Thus, dip-coating can be used for a rapid upper estimation of the ZnO-PEDOT:PSS barrier heights for developing inkjet printable optoelectronic sensors. When using low concentrated PEDOT:PSS suspensions, we observed negative photoresponse of the junction; this is consistent with the formation of a $\mathrm{ZnO}$ NPs and PEDOT:PSS nanocomposite which we obtained on porous semi-transparent ZnO-NP films.
\end{abstract}

Keywords: photosensor, printed electronics, inkjet printing, electrophoretic deposition, heterojunction, negative photoresponse

\section{INTRODUCTION}

Printed electronics is strongly increasing in the field of photonics, as inkjet printing is of interest for roll-to-roll or sheet-to sheet fabrication of flexible optoelectronic devices and flexible organic photovoltaics. Inkjet printing techniques, and in general solution based coating techniques, are gaining a revival with the development of organic semiconductor inks, such as PEDOT:PSS (poly(3,4-ethylenedioxythiophene) polystyrene sulfonate), and solution processable inorganic nanomaterials, like metal and metal oxide nanoparticle suspensions.

Devices, such as large area flexible photovoltaic cells have been demonstrated. Furthermore, the field for flexible robotics, drones, and collaborative robotics for industrial automation is emerging and requires novel flexible low weight sensor solutions. Basic inkjet printable photosensitive devices can be fabricated based on the heterojunction between organic and inorganic semiconductors, such as ZnO-PEDOT:PSS [1],[2],[3], where PEDOT:PSS plays the role of a hole conductor (p-type) and $\mathrm{ZnO}$ the role of an n-type semiconductor. Well defined PEDOT:PSS/ZnO layered structures and printed devices, as well as ZnO-PEDOT:PSS blended layers have been investigated by several research groups, see e.g. [4] and references therein. However, the development of devices by inkjet printing needs printhead adjustments and is therefore time demanding. The question arises thus, whether techniques such as drop-casting and dip-coating, can be used for rapid prototyping and evaluation of heterojunctions in order to support the development of inkjet-printable flexible optoelectronic sensors.

In this paper, we compare three different deposition methods of PEDOT:PSS on ZnO layers and determine resulting barrier heights from electrical characterization. For heterojunctions made from low concentration suspensions of PEDOT:PSS and deposited on porous $\mathrm{ZnO}$, we find negative photoresponse.

\section{EXPERIMENTAL}

\subsection{Materials, heterojunction fabrication, and measurements}

For the fabrication of thin flexible optoelectronic devices, we use ITO covered polyethylene terephthalate (PET) foils (nominal sheet resistance $100 \Omega$ /sq, Sigma Aldrich) as substrates. Zinc oxide nanoparticles (ZnO-NPs) are synthesized according to Pourrahimi et al. [5] using zinc acetate as precursor. Their hydrodynamic diameter ranged between 200 to $300 \mathrm{~nm}$ (in intensity) as analysed by dynamic light scattering (DLS) (zetasizer nanoZS, Malvern). ZnO-NPs are then deposited from aqueous suspension $(1 \mathrm{~g} / \mathrm{L})$ by electrophoretic deposition $(-1.0 \mathrm{~V}$, $15 \mathrm{~min}$ ) on ITO/PET cathode using a platinum counter electrode. The sheet resistance of the obtained 
$\mathrm{ZnO} / \mathrm{ITO} / \mathrm{PET}$ samples $(18 \pm 1 \Omega / \mathrm{sq})$ is measured using a collinear four-point probing sensor. We use PEDOT:PSS (poly(3,4-ethylenedioxythiophene) polystyrene sulfonate) PH1000 (Clevios-Heraeus) as hole conductor for the organic-inorganic heterojunction with $\mathrm{ZnO}$. For dip-coating and printing, the $\mathrm{pH}$ of PEDOT:PSS solution is adjusted to $\mathrm{pH}=7.5$ with $\mathrm{NaOH}(01 \mathrm{M})$ to avoid the dissolution of $\mathrm{ZnO}$. Dip-coating is performed with an automated dip-coater (Riegler \& Kirstein $\mathrm{GmbH}$ ). Per sample, a total of 3 to 5 layers is deposited $(5 \mathrm{~min}$ immersion, immersion and retraction speed of $0.1 \mathrm{~cm} / \mathrm{s}$ and drying in air after each deposited layer). For drop casting, small droplets are deposited using a laboratory micropipette. Inkjet printing is performed with glass capillaries (inner nozzle diameter $60 \mu \mathrm{m}$ ) using a SonoPlot Microplotter II (PEDOT:PSS $1 \mathrm{~g} / \mathrm{L}, \mathrm{pH}=8$, filtered with a $1.2 \mu \mathrm{m}$ pore size membrane prior to printing in order to avoid nozzle clogging).

For electrical characterization, miBots and a Keithley2401 source meter with LabView interface are used for recording current voltage (I-V) curves. Layer thickness, topography, and roughness have been inspected by optical interferometry (Filmetrics 3D Profilometer) and atomic force microscopy (AFM, Park XE7).

\subsection{ZnO-PEDOT:PSS heterojuction analysis}

For the analysis of the ZnO-PEDOT:PSS heterojunction, the recorded I-V curves are analysed along the adapted model of thermionic emission describing the behaviour of practical Schottky barriers (Eq. (1)) [6]. For applied voltages $>25 \mathrm{mV}(=3 \mathrm{kT} / \mathrm{q})$ at ambient temperature the voltage drop is modelled as:

$$
I=I_{S} \exp \left(q\left(V-I R_{s}\right) / n k T\right) \text { with } I_{S}=A_{e f f} A^{* *} T^{2} \exp \left(-q \Phi_{B} / n k T\right)
$$

where $\mathrm{q}$ is the electronic charge, $V_{D}=V-I R_{S}$ the applied voltage (with $V$ the voltage drop across the junction, $R_{S}$ the series resistance), $A_{\text {eff }}$ the effective area of the junction, $A^{* *}$ the effective Richardson constant, $\Phi_{B}$ the barrier height, $n$ the ideality factor, $k$ the Boltzmann constant, and $T$ the absolute temperature. We use an effective Richardson constant of $\mathrm{A}^{* *}=36 \mathrm{~A} \cdot \mathrm{cm}^{-2} \mathrm{~K}^{-2}$ [7] and apply data analysis according to Cheung and Cheung [6], as well as to Norde et al. [8] for extracting the values of barrier height, ideality factor, and series resistance.

\section{RESULTS and DISCUSSION}

\subsection{Dip coating, inkjet printing, drop casting}

Figure 1 shows the device architecture (Fig. 1a) and examples of ZnO-PEDOT:PSS heterojunction samples as prepared by dip-coating (Fig. 1b,1c), inkjet printing (Fig. 1d) and drop casting (Fig. 1e). We experimentally verified that any measured non-linear I-V behaviour comes only from the ZnO-PEDOT:PSS junction. The use of DMSO as additive improves the homogeneity of PEDOT:PSS distribution in the drops (Fig. 1e). For this study, we have however decided to investigate the ZnO-PEDOT:PSS heterojunction without additives, except $\mathrm{NaOH}$ for $\mathrm{pH}$ adjustment.
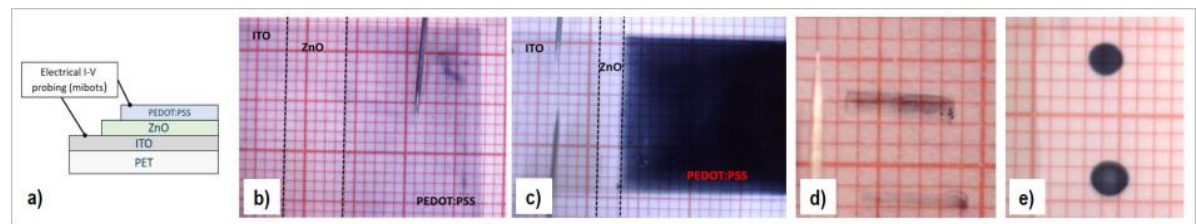

Figure 1: a) ITO/ZnO/PEDOT:PSS heterojunction devices architecture; examples of PEDOT:PSS coating obtained by b) dip coating (1 g/L, 5 layers $), c)$ dip-coating (10 g/L, 3 layers $), d)$ inkjet printing ( $1 \mathrm{~g} / \mathrm{L}$, bottom: single printed, top: double printed), e) drop casting (bottom: $1 \mathrm{~g} / \mathrm{L}$, top: $1 \mathrm{~g} / \mathrm{L}$ with 5\% DMSO additive).

\subsection{Analysis of the deposition and junction properties}

Figure 2 shows examples of measured topography data. ZnO layers from electrophoretic deposition generally exhibit low surface roughness $(R a=1.27 \mathrm{~nm})$; a $\mathrm{ZnO}$ film thickness of at least $70 \mathrm{~nm}$ is found.
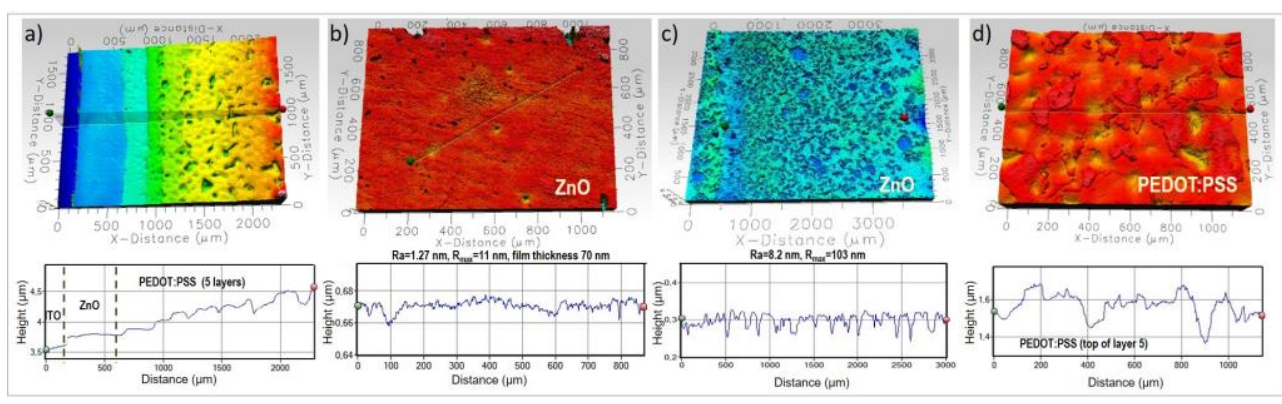

Figure 2: Profilometer data and roughness analysis: a) example of a heterojunction device from dip-coating, $b$ )

$\mathrm{ZnO}$ layer of a) $\left.\left(R a=1.27 \mathrm{~nm}, R_{\max }=11 \mathrm{~nm}\right), \mathrm{c}\right)$ example of a porous rough $\mathrm{ZnO}$ layer $(R a=8.2 \mathrm{~nm}$, $R_{\max }=103 \mathrm{~nm}$ ), d) top PEDOT:PSS layer of a). 
Some $\mathrm{ZnO}$ film zones exhibit pronounced porosity and roughness ( $R a=8.2 \mathrm{~nm}$, maximum sample peak to valley height $R_{\max }=103 \mathrm{~nm}$ ), e.g. Fig. 2c. Atomic force microscopy data (scan size $5 \mu \mathrm{m} \times 5 \mu \mathrm{m}$, not shown here), furthermore reveal nanoscale roughness of typically $40 \mathrm{~nm} \pm 10 \mathrm{~nm}$ and show the $\mathrm{ZnO}$ particles within the granular structure of the films. Dip-coating leads to PEDOT:PSS layers as observed in Fig. 2a,d. For 5 layers the (optical) surface roughness is $R a=11.5 \mathrm{~nm}\left(R_{\max }=88.5 \mathrm{~nm}\right)$ (Fig. 2e); lateral topographic feature sizes increase upon deposition of thick PEDOT:PSS films (Fig. 2d). Note that the samples have not been annealed.

Fig. 3 shows examples obtained from inkjet printing and drop-casting. Inkjet printing fills the porous structure in $\mathrm{ZnO}$ (Fig. 3a), leading to smooth printed lines of about $130 \mathrm{~nm}$ height. The observed linewidths (about 130$150 \mu \mathrm{m})$ are consistent with the used nozzle diameter $(60 \mu \mathrm{m})$. For drop casting of PEDOT:PSS without further additives, the profile shows the coffee-stain effect (Fig 3b), as also seen in Fig. 1d.
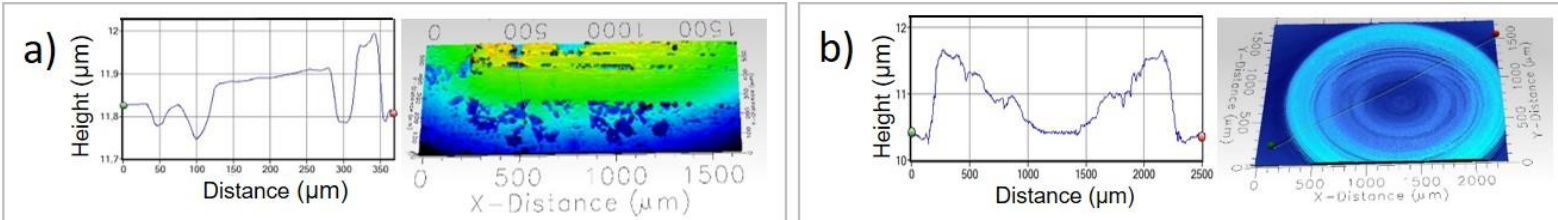

Figure 3: Examples of PEDOT:PSS on ZnO/ITO/PET (profilometer data) as obtained from a) inkjet printing, $b$ ) drop casting.

Fig. 4a shows I-V curves as measured on dip-coated samples. Using 10 g/L PEDOT:PSS, very similar I-V characteristics with a slight rectifying behaviour are obtained for 3 layers and for 5 layers, indicating that the heterojunction is established for 3 layers. Depositing diluted PEDOT:PSS (1 g/L) in comparison, leads to a clearly pronounced rectifying behaviour and higher current densities at the same applied bias voltage (Fig 4a). The distance chosen between electrical probes (typically $23 \mathrm{~mm}$ ) and the active surface area were similar $\left((2.6 \pm 0.1) \mathrm{cm}^{2}\right)$ for all dip-coated samples. Diluted PEDOT-PSS $(1 \mathrm{~g} / \mathrm{L})$ shows modified viscosity and wetting behaviour, resulting in a smoother film surface and thinner layers, thus favouring intermixing with the $\mathrm{ZnO}$ nanoporous structure and improving interface formation between $\mathrm{ZnO}$ and PEDOT:PSS.

Indeed, for PEDOT:PSS layers prepared from low concentration we observed either a low or even a negative photoresponse under light exposure with an office table halogen lamp (Fig. 4b), which we did not observe with the high concentration $(10 \mathrm{~g} / \mathrm{L})$ PEDOT:PSS leading to a film thickness significantly thicker than the ZnO layer. Observation of negative photoresponse is consistent with $\mathrm{ZnO}$ nanoparticle embedding in PEDOT:PSS as this can lead to charge trapping in PEDOT:PSS around ZnO NPs [4]. We thus conclude that the deposition of the diluted PEDOT:PSS favours intermixing of the polymer with the porous oxide (Fig. 2c, 3a) in a suitable ratio for embedding $\mathrm{ZnO}$ in PEDOT:PSS. This result opens perspectives for printable organic-inorganic heterojunctions with adjustable negative photoresponse. Further investigation of nanocomposite layer formation in coating and printing of NP-ZnO and PEDOT-PSS will be needed to develop materials processing and inks for devices with negative photoresponse.
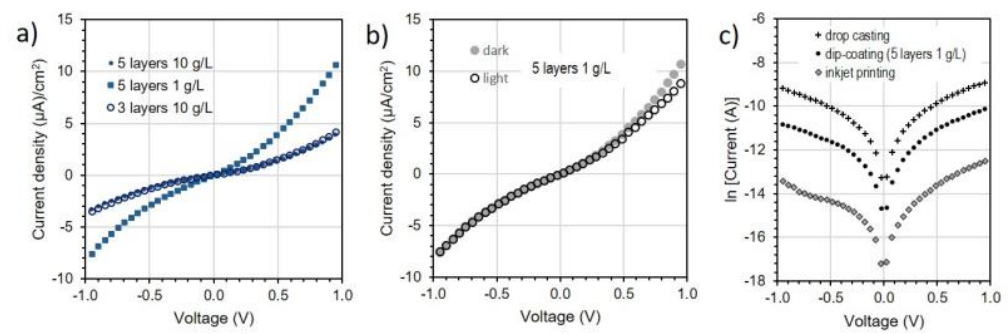

Fig 4: Electrical characterisation of ZnO-PEDOT:PSS/ITO/PET devices: a) I-V characteristics, b) example of negative photoresponse, $c$ ) In(current) vs V plots: similar behaviour is observed for all three deposition methods.

For the further analysis of I-V curves of ZnO-PEDOT:PSS junctions, we apply the method of Cheung and Cheung [6], and Norde et al. [8]. We adopt the notations of the relevant equations of the functions $H(I)$ and $F(V)$ as denoted by Ahmad et al. [9].
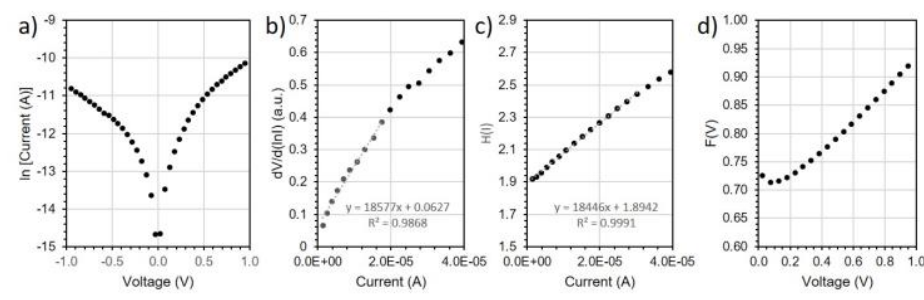

Figure 5.: Examples of the analysis of I-V curves for the extraction of barrier heights, ideality factor, and series resistance according to the methods of Cheung and Cheung $(a-c)$, as well as Norde $(d)$. Data shown here, example for 3 layers of $10 \mathrm{~g} / \mathrm{L}$ PEDOT:PSS ( $\mathrm{pH} 7.5$, dip-coating). Values of $\Phi_{B}, n$, and $R_{s}$ are listed in Table 1. 
Fig. 5 shows illustrates the analysis of I-V curves: $\ln I$ vs $V$ curves for forward and backward bias show the rectifying behaviour (Fig 5a); $d V / d(\operatorname{lnI}) v s$. I graphs (Fig. 5b) allow to determine the series resistance, $R_{s}$, (from the slope) and the ideality factor, $n$, (from the intercept taking into account the temperature); the $H(I)$ plots (with $H(I)=I R_{s}-n \Phi_{B}$ ) (Fig. 5c) also allow to determine $R_{s}$ (from the slope), as well as $\Phi_{B}$ (from the intercept taking into account the ideality factor). From $F(V)$ [8], (Fig. 5d), we also extract values for $\Phi_{B}$ and $R_{s}$.

Table 1 summarizes the values obtained from analysis on dip-coated, inkjet printed and drop casted devices.

Table 1: Extracted parameters from the analysis of I-V curves

\begin{tabular}{|l|r|r|c|c|c|c|c|}
\hline \multicolumn{2}{|l|}{ Junction model } & \multicolumn{3}{|c|}{ Cheung and Cheung [6] } & \multicolumn{3}{c|}{ Norde [8] } \\
\hline Parameter & $\begin{array}{c}\Phi_{\mathrm{B}} \\
(\mathrm{eV})\end{array}$ & $n$ & $\begin{array}{c}\mathrm{R}_{\mathrm{s}}(\mathrm{k} \Omega) \\
\text { arom } d V / d(\ln I), \\
{ }^{\mathrm{b}} \text { from } H(I)\end{array}$ & $\begin{array}{c}\Phi_{\mathrm{B}} \\
(\mathrm{eV})\end{array}$ & $\begin{array}{c}R_{s}(\mathrm{k} \Omega) \\
\text { for } \gamma=\gamma_{\min }>\mathrm{n}\end{array}$ & $\begin{array}{c}R_{s}(\mathrm{k} \Omega) \\
\text { for } \gamma=10\end{array}$ \\
\hline \multirow{3}{*}{ Dip-coating } & 3 layers 10g/L & 0.77 & 2.5 & $18.6^{\mathrm{a}}, 18.4^{\mathrm{b}}$ & 0.70 & $39.1(\gamma=3)$ & 17.8 \\
\cline { 2 - 9 } & 5 layers 10g/L & 0.78 & 4.1 & $57.1^{\mathrm{a}}, 58.0^{\mathrm{b}}$ & 0.73 & $30.2(\gamma=5)$ & 58.6 \\
\cline { 2 - 9 } & 5 layers 1g/L & 0.78 & 1.6 & $37.2^{\mathrm{a}}, 37.4^{\mathrm{b}}$ & 0.75 & $9.7(\gamma=2)$ & 35.7 \\
\hline Inkjet & $1 \mathrm{~g} / \mathrm{L}$ & 0.52 & 2.9 & $203^{\mathrm{a}}, 204^{\mathrm{b}}$ & 0.67 & $19.2(\gamma=3)$ & 174 \\
\hline Drop-casting & $10 \mathrm{~g} / \mathrm{L}$ & 0.65 & 2.7 & $5.8^{\mathrm{a}}, 5.6^{\mathrm{b}}$ & 0.57 & $1.3(\gamma=3)$ & 6.3 \\
\hline
\end{tabular}

For the dip-coated films, the barrier heights obtained by the Cheung \& Cheung method are in good agreement with $0.8 \mathrm{eV}$ reported by Nagata et al. [10] for the PEDOT:PSS/ZnO interface. Our linear regressions according to the method by Cheung \& Cheung show consistency between both values of series resistance. The absolute value of series resistance depends also on the choice of the probe positions on the samples, e.g. due to limited conductivity of the ITO electrode. Further investigations with PEDOT:PSS suspensions optimized for deposition by inkjet printing shall further elucidate the influence of surface roughness and nanocomposite formation for drop casted and inkjet printed heterojunctions between porous $\mathrm{ZnO}-\mathrm{NP}$ films and PEDOT:PSS.

\section{CONCLUSIONS}

In summary, we have investigated solution-based processing and electrical behaviour of ZnO-PEDOT:PSS heterojunctions. Barrier heights obtained from dip-coated samples are in agreement with literature and provide an upper limit compared to inkjet printed and drop casted devices. Furthermore, we have found negative photoresponse for the deposition of PEDOT:PSS suspensions of low concentration on our porous nanoparticle $\mathrm{ZnO}$ films prepared by electrophoretic deposition. This result is consistent with intermixing and embedding of ZnO-NPs by PEDOT:PSS and opens perspectives for the development of flexible optoelectronic devices with negative photoresponse.

\section{ACKNOWLEDGEMENTS}

This work has been financially supported by INTERREG, Communauté du Savoir France-Suisse, project MACAFLEX 4.0.

\section{REFERENCES}

[1] B. Sharma, N. Khare, and S. Ahmad: A ZnO/PEDOT:PSS based inorganic/organic heterojunction, Solid State Communications, vol. 149, pp. 771-774, 2009.

[2] Nikolay Kurtev, Slavka Tzanova, and Silvia Schintke: Photodetector based on electrochemically deposited $\mathrm{ZnO}$ and inkjet printed PEDOT:PSS heterojunction, Electrotechnica \& Electronica, vol. 50 (7-8), pp. 1418, 2015, ISSN 0861-4717.

[3] N. Hernandez-Como et al.: Ultraviolet photodetectors based on low temperature processed ZnO/PEDOT:PSS Schottky barrier diodes, Mater. Sci. Semicon. Proc., vol. 37, pp. 14-18, 2015.

[4] G. Harikrishnan et al.: Negative photoresponse in ZnO-PEDOT:PSS nanocomposotes and photogating effect, Nanoscale Adv. 1, 2435-2443 (2019).

[5] A. M. Pourrahimi et al.: Water-based synthesis and cleaning methods for high purity ZnO nanoparticlescomparing acetate, chloride, sulphate and nitrate zinc salt precursors, RSC Adv., Vol. 4, 35568, 2014.

[6] S.K. Cheung and N.W. Cheung, Extraction of Schottky diode parameters from forward current-voltage characteristics, Appl. Phys. Lett., vol. 49, pp. 85-87, 1986.

[7] F. E. Jones, B.P. Wood, J.A. Myers, C.D. Hafer, and M.C. Lonergan: J. Appl. Phys, vol 86, pp. $6431,1999$.

[8] H. Norde, A modified forward I-V plot for Schottky diodes with high series resistance, J. Appl. Phys. Vol. 50, p. 5052, 1979.

[9] Z. Ahmad and M.H. Sayyad, Extraction of electronic parameters of Schottky diode based on anorganic semiconductor methyl-red, Physica E, vol. 41, pp 631-634, 2009.

[10] T. Nagata et al.: Hard X-ray photoelectron spectroscopy study on band alignment at poly (3,4ethylenedioxythiophene):poly(styrenesulfonate)/ZnO interface, Appl. Phys. Lett., vol. 117, 244501, 2012. 